\title{
Écrire le travail : la position de l'auteur les sujets de son écriture, les destinataires en questions. Le cas des comptes rendus des réunions des cercles de qualité.
}

Writing about work: the writer's posture. The themes of his writings - The

recipents. The case of the minutes of " cercles de qualité " meetings.

\section{Bernard Buron}

\section{(2) OpenEdition}

12 Journals

Édition électronique

URL : http://journals.openedition.org/edc/2488

DOI : $10.4000 /$ edc. 2488

ISSN : 2101-0366

Éditeur

Université Lille-3

\section{Édition imprimée}

Date de publication : 1 janvier 1995

Pagination : 13-39

ISSN : 1270-6841

\section{Référence électronique}

Bernard Buron, «Écrire le travail : la position de l'auteur les sujets de son écriture, les destinataires en questions. Le cas des comptes rendus des réunions des cercles de qualité. », Études de communication [En ligne], 16 | 1995, mis en ligne le 21 juin 2011, consulté le 30 avril 2019. URL : http://

journals.openedition.org/edc/2488; DOI : 10.4000/edc.2488

Ce document a été généré automatiquement le 30 avril 2019

(c) Tous droits réservés 


\section{Écrire le travail : la position de l'auteur les sujets de son écriture, les destinataires en questions. Le cas des comptes rendus des réunions des cercles de qualité.}

Writing about work: the writer's posture. The themes of his writings - The recipents. The case of the minutes of " cercles de qualité » meetings.

\section{Bernard Buron}

Les procédures de certification des entreprises peuvent se synthétiser en trois phases sur le plan de l'écriture du travail. Dans un premier temps, les procédures réellement mises en œuvre sont enregistrées par écrit, puis elles sont rationalisées pour correspondre à des critères définis d'efficacité et de rentabilité. Dans un second temps, ces nouvelles procédures, élaborées à partir du travail réel - du moins, tel qu'il a été écrit«reviennent » dans les ateliers et les services d'où elles sont issues et deviennent les nouvelles prescriptions. Enfin, le contrôle de ces prescriptions est réalisé par écrit ${ }^{1}$. Ce mouvement touche aussi bien la fonction publique d'état et territoriale que les entreprises privées; dans ces dernières, les secteurs de production sont concernés au même titre que les services fonctionnels. On aurait sans doute tort de considérer qu'il s'agit seulement là d'un nouvel avatar du taylorisme. Si l'objectif déclaré est analogue ${ }^{2}$ - rationaliser le travail pour augmenter la productivité et la qualité des produits et des services - les modalités concrètes de cette rationalisation sont très différentes. Dans tous les cas, les salariés, quelque soit leur qualification sont " associés " à ces procédures : ils participent aux différentes phases de l'écriture du travail et des procédures réels; de la modification de ces procédures écrites et de l'écriture des nouvelles ; enfin, ils écrivent la manière dont les procédures modifiées sont mises en œuvre au quotidien. 
2 Dans ce texte, on se propose d'analyser les problèmes que pose l'écriture du travail et des procédures à partir de l'exemple des cercles de qualité qui ont été en vogue dans les entreprises tout au long de la dernière décennie. Si les cercles de qualité ont régressé ou disparu depuis, ils permettent pourtant de montrer une partie des enjeux sociaux réels qui se négocient autour de ce type d'écrits professionnels.

3 La première question qui peut être posée est celle de l'auteur de ces écrits professionnels. Si l'on se plait à penser dans notre société que l'écriture est toujours singulière, elle résulte au minimum, dans le cas qui nous occupe, d'une énonciation plurielle. Cette question est multiforme. Elle se pose au plan juridique: en terme de responsabilité de l'auteur de l'écrit professionnel - dans les procédures qualité qui se développent l'écrit peut servir de preuve ${ }^{4}$; en terme de droit d'auteur et de reconnaissance de la propriété intellectuelle de l'écrit ${ }^{5}$. Elle se pose au plan de la formation des travailleurs à l'écrit et de la reconnaissance de ces nouvelles capacités requises en termes de qualification, de statut et de salaire.

4 De la même manière, le sujet sur lequel on écrit est à questionner à l'aune du processus dans lequel l'écriture du travail et des procédures prennent leur place. L'analyse des comptes rendus des réunions dans lesquelles les salariés énoncent leurs savoirs (Buron 1989) est pertinente car elle permet d'appréhender les écarts entre ce qu'ils disent de leur travail réel et des procédures en vigueur et ce qu'ils en écrivent, compte tenu de leur position dans l'entreprise et des enjeux liés à leur position. Si on arrive à saisir les écarts entre faire (quelque chose), dire ce que l'on fait et écrire ce que l'on dit faire ${ }^{6}$, on montre les limites de la conception de la qualité qui prévaut aujourd'hui et qui tend à réduire l'écriture des procédures et de leur application à une simple opération technique, sans voir la multitude et la variété des enjeux sociaux qui s'y négocient.

5 Avant d'aller plus loin, précisons un point. Si l'écriture au travail (et du travail), telle que nous venons de la définir cursivement, se développe d'une manière qui semble attestée, on doit prendre garde à l'illusion de la nouveauté. En effet, ce n'est pas d'aujourd'hui que l'écrit est mobilisé au travail. Depuis la Révolution industrielle, et en particulier avec le développement de théories d'organisation du travail telles le Taylorisme, on écrit au travail.

6 Si la question de l'écriture au travail, de l'auteur et du sujet sur lequel il écrit, devient pertinente, c'est d'abord, et peut-être surtout, parce que de nouvelles catégories de salariés sont concernées. Ce qui est en jeu ne relève pas d'un développement linéaire, et inéluctable, des «besoins " des entreprises en termes de savoirs requis et mobilisés au travail, et/ou de niveau de connaissances et de formation des salariés, qui se manifesterait par leur accès généralisé à l'écriture dans le travail. Le phénomène qui est en cause est celui de la transformation de la configuration ${ }^{7}$ des entreprises, dans laquelle de nouvelles catégories utilisent l'écrit comme moyen de travail. On pourrait, dans une certaine mesure, dire que ces nouvelles catégories " accèdent » à l'écriture dans le cadre du travail, alors que cela leur était souvent interdit, de fait, précédemment, mais il nous semble plus pertinent de poser que les formes actuelles d'organisation de la production requièrent la capacité des salariés à produire des écrits relatifs à leur travail. Ce sont les principes et les modalités de la connaissance, de la transmission et du contrôle du travail, pertinent pour un groupe social particulier dans les entreprises qui semblent être proposés ou imposés à tous. 
7 Cet effort de construction et de distanciation théoriques nous semble d'autant plus utile que la question de l'écrit au travail interroge fortement le rapport du chercheur à son propre travail et à son écriture. Dans le milieu intellectuel, l'écriture est souvent considérée comme une expression de l'individualité et de la liberté de l'auteur, en même temps elle est un moyen de manifester sa maîtrise professionnelle et une condition de sa reconnaissance dans le milieu. Sans cet effort, on risquerait de projeter un rapport spécifique à l'écriture sur l'ensemble des écritures et des rapports sociaux à l'écriture, c'est-à-dire de considérer l'écriture comme liberté8.

\section{L'analyse des écrits professionnels : questions multiples}

8 L'analyse des écrits professionnels nous semble pouvoir intégrer les acquis de la sociologie des champs, telle que Pierre Bourdieu l'a développée, et en particulier la sociologie du champ littéraire (Bourdieu, 1980 ; 1994). De ce point de vue, on peut donc considérer les écrits professionnels comme des «œuvres » spécifiques produites par des auteurs spécifiques qui occupent une position dans un champ spécifique ${ }^{9}$ de production d'écrits relatifs au travail et aux procédures dans les entreprises. Cette approche nous semble pertinente dans le cas des écrits produits par les cercles de qualité car tous les participants sont des volontaires, et donc, d'une certaine manière, acceptent de jouer le jeu, considèrent «que le jeu vaut la peine d'être joué, que le jeu en vaut la chandelle». L'approche en terme de champ n'exclut pas les conflits entre les « joueurs », bien qu'il y ait accord sur le jeu et sur le fait d'y participer elle permet même de penser que les affrontements dans le champ tiennent aussi à cet accord sur le jeu.

9 Si on adopte cette approche, cela conduit à objectiver la structuration du champ de production d'écrits professionnels dans l'entreprise et la position relative des différents textes ou "œuvres» qui y sont produits ${ }^{10}:$ le règlement intérieur, les différentes procédures techniques, les définitions des postes, des responsabilités...

10 On doit ensuite analyser la position de l'auteur de l'écrit professionnel dans ce champ (dans le cas qui nous intéresse, le compte rendu des réunions des cercles de qualité) et la légitimité qui est accordée à l'auteur et à la position qu'il occupe dans le champ de production d'écrits professionnels où il écrit.

11 Enfin, en dernier lieu, il convient d'analyser ce que l'auteur écrit, les sujets sur lesquels il écrit et la manière dont il écrit, par rapport à ce qui pourrait s'écrire. Cela permet d'appréhender, d'une part, les sujets sur lesquels l'auteur considère légitime d'écrire par rapport à la position qu'il occupe dans le champ. D'autre part, on peut saisir la représentation qu'il a du champ, c'est-à-dire la représentation qu'il a de sa position et des enjeux liés à l'écriture par rapport aux autres positions dans le champ de production d'écrits professionnels.

12 La théorie des champs est intéressante pour analyser les enjeux qui se négocient dans les écrits professionnels, en fonction des auteurs et de leur position dans le champ, des sujets sur lesquels ils écrivent et de la manière dont ils écrivent. Il convient pourtant de ne pas autonomiser ce champ par rapport aux autres champs qui se croisent dans l'entreprise. Si on peut considérer qu'il y a dans chaque entreprise un champ de production d'écrits professionnels, aucune ne se réduit à ce seul champ. 

nouvelles catégories de salariés "accèdent " à l'écriture. On doit aussi prendre en compte les caractéristiques de la "raison graphique», de l'écriture en tant que phénomène social et culturel spécifique. Jack Goody (1977) montre que le passage à l'écriture dans une société transforme la représentation du temps : linéaire et continu dans les sociétés sans écriture, il devient réversible et discontinu dans les sociétés avec écriture. Ce qui nous intéresse ici, c'est de penser la transformation que produit l'introduction de l'écriture au travail pour ces catégories dont on requiert aujourd'hui la capacité à produire des écrits relatifs à leur travail.

Placées en situation de produire des règles écrites, de procédures, de contrôle du travail, etc... ou, tout au moins, associés à leur production et à leur écriture, ces catégories vivent la contradiction entre Thémis et Mètis, entre la règle intangible et l'intelligence rusée de la situation. Mètis et Thémis ne s'opposent pas seulement dans leurs principes profonds, elles s'opposent concrètement dans l'entreprise par le fait qu'elles sont classiquement portées et représentées par des groupes différents: ceux qui produisent règles et procédures de travail et ceux qui les appliquent en les détournant ne sont pas les mêmes. d'écriture du travail ont des principes de réalité identiques. Dans la mythologie grecque, la parole de Thémis

"a une valeur assertorique ou catégorique, elle énonce le futur comme s'il était déjà écrit ; exprimant ce qui sera sur le mode de ce qui est, (...) elle ordonne ou elle interdit. "

Elle marque

« les frontières à ne pas franchir, les préséances à respecter pour que chacun soit à jamais maintenu dans les limites de son domaine et de son rang. »

Thémis présuppose

« un ordre déjà instauré, définitivement fixé et établi. »

\section{À l'inverse}

«Mètis intervient quand le monde divin apparaît encore en mouvement ou que l'équilibre de ses forces se trouve momentanément rompu (...) pour triompher [il faut] faire preuve, en même temps que d'audace et de force, d'initiative intelligente et d'esprit d'invention. » (Détienne et Vernant, 1974).

Les nouvelles catégories qui accèdent à l'écriture au et du travail dans les "démarches qualité » comme dans les procédures de certification, sont durant la grande majorité de leur temps de travail du côté de Mètis, de la pratique, c'est-à-dire du détournement de la règle formelle ${ }^{11}$ pour atteindre les objectifs de qualité imposés. Le détournement permet de répondre aux variations des conditions du travail, qui, quelque soit le niveau d'automatisation, ne sont jamais constantes. Le détournement n'est possible que s'il y a règle, la règle n'est efficace qu'à la condition de la détourner ${ }^{12}$.

L'analyse des écrits professionnels implique de ne pas autonomiser ces œuvres (ou textes) du champ de production d'écrits professionnels (ou contexte) dans lequel ils sont produits et où ils font sens. On doit donc considérer que l'entreprise est aussi un lieu de conflits dans lequel travaillent, collaborent et s'affrontent des groupes qui ont des positions et des intérêts différents, des représentations concurrentes de leur place et de leur avenir. Ces groupes s'affrontent aussi pour définir ce qu'il est légitime de faire ou de ne pas faire, pour imposer leur vision légitime des choses. Dans le cas qui nous occupe ici, faire écrire les règles et procédures par les travailleurs qui les appliquent, ou, au moins 
les associer à cette écriture, c'est aussi lutter pour imposer une vision du monde, peutêtre plus proche de Thémis que de Mètis.

\section{2 . Le cercle : une position d'écriture dans l'entreprise} plusieurs médiations entre la réalité qu'ils vivent au quotidien sur leur poste de travail dans l'atelier ou le bureau. Ces différentes médiations permettront l'écriture. La réunion marque un retrait de l'espace quotidien des participants et de sa hiérarchie; la prise de parole sur cet espace familier dans un espace qui ne l'est pas. L'écriture du compte rendu se fait par retrait de l'espace-temps du cercle, par sélection de ce qui mérite d'être écrit, c'est-à-dire porté à la connaissance des autres, les pairs et la hiérarchie.

\section{Les moyens de travail de cette position d'écriture}

Les principaux moyens de travail de cette position d'écriture visent à faire émerger des sujets d'écriture. Invités «à remettre [leur] cerveau en marche» (De Ligny, 1982, p. 32), les membres du cercle utilisent un outil, le brainstorming ${ }^{15}$ qui " permet à chacun de s'exprimer, d'exprimer toutes ses idées, sans restriction et sans contraintes » (Raveleau, 
1983, p. 160). Cet outil est utilisé pour recenser les dysfonctionnements de l'atelier et pour imaginer les solutions possibles au problème étudié. Durant ce moment particulier, la parole doit être totalement libre, chacun énonce spontanément et sans réfléchir une idée ou une proposition ${ }^{16}$. La cohérence logique de l'idée proposée est moins valorisée que la proposition elle-même, le fait qu'elle s'énonce.

Après ce temps d'énonciation des sujets potentiels à étudier, et sur lesquels écrire, vient le temps du choix entre cette pluralité. Ici aussi, un outil ${ }^{17}$ est proposé : le vote pondéré. Chacun des membres vote en attribuant un nombre de points à chaque problème recensé, par exemple compris entre un et cinq par ordre d'importance croissante. Celui qui obtient le plus de points est considéré comme le plus important pour le groupe. Il pourra devenir un sujet d'écriture.

Après chaque réunion, le cercle établit un compte rendu de l'avancement de son activité. Cette communication écrite a deux destinataires explicitement désignés : la hiérarchie de l'entreprise (comité de pilotage, coordonnateur, mais aussi hiérarchie de l'atelier); les collègues de l'atelier ou du service. Cet écrit professionnel a un double objectif explicite : contrôler et coordonner l'activité des différents cercles, le choix de leurs sujets ${ }^{18}$; intégrer le cercle dans son atelier. Il s'agit de rendre visibles et lisibles son activité et les améliorations produites, ce qui est censé entrainer l'adhésion de ceux qui ne participent pas et susciter leur désir de participer. Le compte rendu est une réponse organisationnelle à l'objectif politique d'intégration de cercles de qualité dans l'entreprise.

Pour analyser les écrits professionnels, il faut pouvoir mettre en relation la structuration du champ de production d'écrits professionnels de l'entreprise, la position institutionnelle de l'auteur et la légitimité que cette position lui donne. Dans le cas qui nous occupe ici, analyser les comptes rendus implique de comprendre ce qui s'écrit, la manière dont on écrit et le sujet sur lequel on écrit, mais aussi les sujets sur lesquels on n'écrit pas. On ne peut comprendre ce dernier point qu'en affinant l'analyse de la position d'écriture qu'est le cercle, en intégrant le ou les destinataires de l'écrit professionnel.

«Avec l'écriture s'instaure nécessairement, à distance de la parole, un lieu d'où peut être appréciée sa conformité : règles du bien-parler (grammaire), du bienpenser (logique), modèles du beau discours (rhétorique) $»^{19}$.

Dans la réalité des rapports sociaux dans l'entreprise, les destinataires « réels » du compte rendu sont au minimum cinq : le comité de pilotage ; la hiérarchie de l'atelier ou du service; les services fonctionnels; les collègues de l'atelier et le groupe lui-même. Tous ont ou peuvent avoir, en fonction du sujet d'écriture, des « règles du bien-parler, du bien-penser », des « modèles du beau discours » différents. Ce qui caractérise la position institutionnelle du cercle, c'est l'obligation, implicite mais parfaitement ressentie, de concilier la pluralité des destinataires et de leurs critères d'appréciation. L'écriture n'est donc possible que si la variété des critères d'appréciation des différents destinataires, réels ou potentiels, est, d'une manière ou d'une autre, prise en compte par l'auteur. De ce fait, les caractéristiques individuelles de celui qui rédige le compte rendu sont secondes par rapport à la position d'écriture. 


\section{S'auto-identifier et se faire reconnaître comme auteur, la condition d'une écriture pour cette position d'écriture}

31 Si le cercle de qualité peut être considéré comme une position d'écriture dans le champ de production d'écrits professionnels de l'entreprise, cette position n'est pas encore occupée par un auteur. On doit donc analyser la manière dont un auteur peut se constituer, occuper cette position, écrire quelque chose et s'intégrer dans l'atelier dont il est issu.

Un cercle est composé de travailleurs qui ont, au minimum, deux points communs : ils sont volontaires pour participer et sont issus du même atelier. Lors de sa première réunion, il rassemble une pluralité de volontaires et n'a pas de capacité collective d'action ou d'écriture. On peut penser avec Denis Segrestin (1980, p. 171-203) que toute action collective implique une communauté de référence minimale, et que l'action collective n'est possible que si le cercle devient, d'une manière ou d'une autre, une forme de référence.

Tout cercle choisit et adopte un nom particulier qui crée un élément commun entre les participants. Ce nom contribue à faire émerger un auteur, en masquant les différences entre les individus rassemblés. Il manifeste l'existence concrète et identifiable de cette position d'écriture, aussi bien pour les membres du cercle que pour les destinataires de cette écriture.

Dans les entreprises observées (Buron, 1992), ce processus de dénomination se déroule de manière semblable. Un brainstorming permet aux membres d'exprimer toutes leurs idées sur le problème posé : trouver un nom pour le cercle auquel ils participent. La technique du vote pondéré permet à un accord de se réaliser sur le nom de l'auteur.

Prenons un exemple. Dans une entreprise de production du secteur militaro-industriel qui fabrique, entre autre chose des parachutes, les trois ouvriers du magasin « matières premières » sont membres du cercle. Le brainstorming fait apparaitre trente noms possibles, le premier tour de vote en laisse dix. Au deuxième tour, l'accord se réalise sur trois noms. Le troisième et dernier tour de scrutin choisit le nom de l'auteur: Les 3 Mousquetons. Les deux autres noms possibles étaient Mag 3 et Magnum 3. Le nom donné à l'auteur n'est ni formel, ni anecdotique, il exprime les représentations que les membres du cercle ont de leur position dans l'entreprise et des enjeux qui se négocient autour de cette position.

Dans cet exemple, on note que le chiffre trois est récurrent, il correspond au nombre d'individus concernés dans le cercle, mais aussi dans l'espace-temps de travail. Les trois noms sur lesquels se réalisait l'accord renvoient à l'entreprise, à son type de production et aux représentations des travailleurs quant à leur position d'écriture et à ses enjeux spécifiques. Avant de se lancer dans le vide, le parachutiste accroche ${ }^{20}$ le système d'ouverture automatique de son parachute grâce à un mousqueton qui représente son assurance, sa sécurité. Pour ses membres, le caractère collectif du cercle et de son action, manifestée par le nom donné à l'auteur, représente aussi une assurance et une sécurité individuelle avant l'épreuve et les dangers qui s'annoncent. Les deux autres noms possibles présentent les mêmes caractéristiques. Mag 3 fait une double référence: la première est directe à magasin, l'espace qu'ils occupent dans la division du travail ; la 
seconde est euphonique à Mach, unité de mesure de la vitesse en aviation (performance). Le troisième choix possible renvoie au double sens de magnum, double bouteille utilisée surtout pour le champagne (convivialité) et calibre d'arme à feu (puissance).

Le cas est identique pour le cercle Boîte à Idées (dans un groupe de la grande distribution). Le vote pondéré donne les résultat suivants :

Boîte à Idées 43 voix; Les idées nouvelles 40 voix; Les 7 mercenaires 40 voix; Le cercle de l'amitié 39 voix; Le groupe de l'amélioration et de la réorganisation 37 voix ; Le renouveau 37 voix ; [puis, toujours en ordre décroissant] Les grosses têtes, Le cerveau, Le perroquet, Les schtroumphettes, (etc.) ${ }^{21}$.

Dans les résultats de ce vote, on voit les traces du jeu entre la position objective et les représentations des membres du cercle. Ce sont des femmes non qualifiées qui, dans une entreprise où le « moral » est mauvais - absentéisme et turn over important - signifient que leurs idées, l'amitié, la réorganisation et leur cerveau changeront les choses. En ce sens, elles "adhérent» au discours et aux objectifs de la direction de l'entreprise. Mais, derrière l'adhésion formelle manifestée, apparaissent une certaine forme d'autodérision ( Le perroquet, Les schtroumphettes) ainsi que la manière particulière dont les travailleuses mobilisées se perçoivent par rapport au collectif de travail de l'atelier (Les 7 mercenaires). Le nom de l'auteur des comptes rendus des cercles de qualité doit donc être analysé comme le produit des tensions qui existent entre la position objective des participants dans la division du travail, les signes d'adhésion au processus d'écriture que la direction les contraint à manifester, les représentations qu'ils ont de leur participation et des enjeux qu'elle implique à l'intérieur de l'atelier.

Il montre que pour ces catégories, dont les « démarches qualité » requièrent les capacités d'écriture, les capacités à produire des écrits sur leur travail et les procédures qu'elles mettent en œuvre, l'écriture au et du travail ne se limite pas à une opération technique. Cette écriture est au contraire toujours sur-investie d'enjeux sociaux majeurs qui la conditionnent, tant dans sa forme que dans son contenu.

\section{Formuler un sujet d'écriture acceptable}

41 L'analyse des écrits professionnels implique une grande proximité du chercheur avec le terrain concret et ses enjeux spécifiques, tels qu'ils sont pensés et vécus par les différents groupes concernés, mais aussi tels qu'ils agissent dans les relations entre les diverses positions en présence. Cette analyse implique la saisie minutieuse des conditions sociales dans lesquelles ces écrits professionnels s'écrivent, la manière dont les sujets d'écriture sont choisis d'abord, écrits ensuite, en fonction de la position de l'auteur dans le champ de production d'écrits professionnels dans l'entreprise.

L'approche proposée ici présente, nous semble-t'-il, l'intérêt d'élargir et d'approfondir le champ de la recherche sur les écrits professionnels. Elle permet d'analyser ce qui, dans les entreprises, est sujet d'écriture pour chaque catégorie d'auteur, située dans une position différente. On peut alors analyser la production de chacune d'elle, montrer comment ce qui s'écrit est écrit. On peut surtout, et alors on élargit la recherche, analyser aussi ce qui ne s'écrit pas, comment tel sujet ne peut pas être sujet d'écriture pour telle catégorie d'auteur. Ainsi, on peut resituer les écrits professionnels dans le champ plus large des rapports sociaux qui dépassent l'entreprise. 
43 Nous proposons un exemple de cette analyse. Le cercle de qualité nommé Oxygène est composé d'employées qualifiées et de techniciennes d'une caisse départementale de la Mutualité Sociale. Le service dont le cercle est issu est chargé de la perception des cotisations et de la gestion des procédures contentieuses engagées contre les assujettis qui ne paient pas, ou qui sont en retard. La procédure contentieuse est jugée inefficace, dysfonctionnelle, par les membres du cercle qui recherchent une nouvelle organisation. Lors de la première réunion qui lui consacrée, ce problème est formulé de la manière suivante : la procédure contentieuse serait défaillante à cause de la 'méconnaissance des procédures contentieux par [les employés du] secteur cotisation"22.

Ainsi, le sujet d'écriture est inacceptable pour, au moins l'un des destinataires réels du compte rendu. Si l'auteur conservait cette formulation, et même si le diagnostic était techniquement juste, elle risquerait de provoquer un conflit entre le cercle et les collègues du secteur cotisation, leurs pairs à qui ils attribueraient la responsabilité du dysfonctionnement. Dans ce cas, ces derniers risqueraient de refuser la solution proposée par le cercle, ce qui entraînerait son échec. On sait que l'intégration des cercles est un objectif politique de la direction, que cette position d'écriture n'a pas le pouvoir d'imposer une de ses solutions mais qu'elle a un "pouvoir de proposition », formellement reconnu par la direction de l'entreprise. Cette position dans la structure hiérarchique contraint l'auteur à rechercher et à obtenir l'adhésion des non membres sur la nouvelle procédure proposée. Dans ce cas, l'auteur ne peut que reformuler le sujet d'écriture de telle sorte que le consensus sur la solution soit possible. Il devient: "les techniciens méconnaissent réciproquement les tâches de chaque unité du service cotisation-contentieux ${ }^{23}$.

La formulation du sujet d'écriture, et sa reformulation jusqu'à ce qu'il soit acceptable par tous les destinataires du compte rendu - collègues de travail, direction de l'entreprise, hiérarchie du service, etc... - demande du temps. Dans le cas décrit, elle a occupé le cercle pendant deux réunions, soit un mois. Le problème étudié concerne maintenant le service tout entier, la méconnaissance des procédures est réciproque, elle est due à l'organisation interne du service et à la division des tâches. Il n'est plus imputé à certains collègues qui dans la nouvelle formulation du problème sont tous appelés techniciens.

Le sujet d'écriture ne se réduit jamais à un énoncé technique qui s'imposerait en vertu d'une objectivité incontestable. Il est toujours conditionné par la position de l'auteur dans la structure hiérarchique et dans le champ de production d'écrits professionnels dans l'entreprise.

$\mathrm{Au}$ point où nous sommes, nous avons un auteur et un sujet d'écriture, qui après un long travail de reformulation est devenu un sujet acceptable par la pluralité des destinataires réels et potentiels de l'écrit professionnel. On pourrait penser que le dysfonctionnement étudié peut être résolu. Tel n'est pas le cas. Nous avons dit plus haut que pour bien comprendre les enjeux qui se jouent autour de l'écriture au et du travail, autour de l'accès à l'écriture, ou de l'accès à de nouveaux sujets d'écriture, pour ces catégories de salariés, il convenait aussi d'analyser ce qui ne s'écrit pas, ce qui ne peut pas s'écrire. Pour comprendre ce qui ne peut pas s'écrire, on doit souvent «sortir» de l'entreprise et des enjeux spécifiques de son champ de production d'écrits professionnels.

Les théories actuelles de la qualité postulent que l'efficacité économique et le consensus social sont réalisés quand les salariés adhèrent à un projet d'entreprise qui pose clairement quelques valeurs simples, incontestables et cohérentes avec l'histoire officielle de l'entreprise. Ainsi, une synthèse harmonieuse se réaliserait entre les nécessités de la 
rigidité de l'organisation pour faire aboutir un projet et les contraintes de flexibilité de l'organisation pour satisfaire à la fois un marché fluctuant et les besoins des salariés.

Dans le cas qui nous occupe, c'est l'adhésion réelle des salariés aux valeurs sociales de l'entreprise qui rend impossible l'écriture d'une nouvelle procédure par les membres du cercle, qui fait que ce dysfonctionnement ne peut pas être un sujet d'écriture, au moins pour l'auteur Oxygène, compte tenu de sa position dans l'entreprise. L'entreprise est membre de la Mutualité française, ses valeurs officielles sont identiques à celles du mouvement mutualiste et, globalement, à celles de tout le système de protection sociale en France. La communication externe de l'entreprise rappelle ces valeurs : la mission de service public de l'entreprise fait obligation de verser l'ensemble des prestations sociales auxquelles chacun des assurés a droit, en vertu de sa situation individuelle particulière. Elle fait aussi obligation d'informer les ayants droit de leurs droits et de veiller à ce que chacun d'eux fasse bien la demande pour tous les droits qui lui sont légalement ouverts ${ }^{24}$. Dans l'entreprise, ces valeurs se concrétisent dans une règle formelle: dans tous les cas, le calcul des prestations doit être fait avec le mode de calcul le plus favorable à l'assuré.

Dans son immense majorité, le personnel adhère aux finalités sociales de l'entreprise, parce qu'elles sont politiquement et humainement valorisées et valorisantes, mais aussi parce qu'une fraction du personnel est issue de la catégorie sociale des bénéficiaires des prestations. Il y a également accord sur la seconde grande dimension de la «mission » de l'entreprise qui consiste à prélever les cotisations sociales sur la fraction active des assurés. Pour le personnel, le prélèvement des cotisations est aussi normal que le versement des prestations. La solidarité entre générations et entre "riches » et « pauvres » rend légitime le versement des prestations et le prélèvement des cotisations.

51 Il y a donc accord sur les valeurs et la mission de l'entreprise, et globalement on peut dire que les règles organisationnelles formelles qu'elles entraînent sont bien acceptées. De la même manière, les objectifs politiques du Conseil d'administration qui tendent à améliorer le service rendu aux assurés en réduisant les délais de versement des prestations sont bien acceptés par le personnel. Ainsi, de manière prototypique, l'entreprise réalise les conditions sociales et politiques que les théories de la qualité considèrent comme idéales: les salariés partagent les valeurs officielles de l'entreprise, ils adhèrent à son projet, etc...

52 Le cercle est situé dans un service qui à la double fonction de percevoir les cotisations et d'engager les procédures contentieuses contre les assujettis qui ne les paient pas ${ }^{25}$. Formellement, le problème est relativement simple : cette procédure doit être engagée contre tout assujetti qui ne paie pas ses cotisations. Dans la pratique, le problème est plus complexe. L'infraction est constatée par le secteur qui perçoit les cotisations, la procédure est conduite par le secteur qui gère les contentieux, mais la responsabilité du déclenchement de la procédure n'est pas clairement définie. Le cercle cherche à résoudre cette indétermination organisationnelle, mais il n'y parvient pas. Les membres du cercle qui travaillent dans le secteur cotisation arguent qu'ils ne sont pas qualifiés pour la procédure contentieuse - ce qui est vrai au plan de la division du travail ; ceux du secteur contentieux arguent qu'ils ne peuvent conduire cette procédure que si elle a été engagée au préalable et qu'ils ne peuvent l'engager car ils ne constatent pas l'infraction. Cette situation rend impossible une écriture sur le sujet. Le cercle abandonne ce sujet d'écriture en l'état parce qu'un consensus sur la solution ne s'est pas dégagé parmi ses membres, et ne se serait sans doute pas non plus manifesté parmi les non membres. La procédure 
contentieuse, pourtant identifiée comme déficiente, reste formellement indéterminée dans son déclenchement.

Il faut chercher les raisons qui ont fait passer ce sujet d'écriture au statut de sujet sur lequel on ne peut pas écrire dans l'adhésion des salariés aux valeurs de l'entreprise, et, en particulier dans la valeur de solidarité. En effet, le secteur d'activité des assujettis est en déclin marqué, ceux qui ne paient pas leurs cotisations se trouvent le plus souvent dans une situation économique très difficile, voire désespérée. Ils sont souvent proches de la retraite, leur revenu est inférieur au SMIC, ils ne pourraient pas trouver un autre emploi. Dans cette situation particulière, appliquer les règles qui, communément, participent au maintien des grandes valeurs abstraites de solidarité de l'entreprise, reviendrait à exercer ces valeurs contre ceux qu'elles sont censées protéger. En raison de leur adhésion à ces valeurs, les salariés refusent que leur secteur engage cette procédure dans la pratique. Aux cotisations, ils acceptent simplement de signaler les cas d'assujettis qui n'ont pas payé leurs cotisations; au contentieux, ils acceptent de gérer des procédures contentieuses qui ont été engagées par un autre service.

L'écriture sur ce sujet se termine par un refus d'écriture. Durant une réunion suivante, les membres du cercle se sont mis d'accord sur plusieurs points. La manière actuelle de procéder doit être conservée, en dépit de son indétermination formelle. Les difficultés qu'elle génère seront résolues au cas par cas, au besoin après discussion entre les salariés du service. D'ailleurs, ces échanges permettront de mieux apprécier la situation réelle des contrevenants. Enfin, et peut-être surtout pour le cas qui nous occupe, cet accord ne doit pas être écrit. Ce refus d'écriture, sur ce sujet, manifeste le conflit entre une règle formelle et l'application juste de ses principes. Une procédure précise entraînerait le déclenchement automatique des poursuites contre les assujettis et priverait les salariés $\mathrm{du}$ droit qu'ils se donnent, au nom des valeurs de l'entreprise ici, d'apprécier les situations au cas par cas.

L'exemple que nous venons de développer montre que l'adhésion du personnel aux valeurs proclamées de l'entreprise ne garantit en rien qu'ils acceptent une rationalisation du travail qui serait nécessaire pour atteindre des objectifs abstraits d'efficacité. Il montre aussi comment un sujet d'écriture, formulé de manière acceptable par les destinataires à un moment donné, peut changer de statut et devenir un sujet sur lequel il est impossible d'écrire pour l'auteur lui-même. Il montre aussi que les refus d'écriture ne peuvent pas être expliqués seulement par un défaut de la formation, de la capacité d'écrire du personnel concerné.

\section{En guise de conclusion}

Nous avons tenté de montrer que les écrits professionnels peuvent être analysés comme des œuvres spécifiques, produites par des auteurs qui occupent une position spécifique dans le champ de production d'écrits professionnels des entreprises. Aujourd'hui, ce champ est à nouveau transformé par les procédures de certification en cours. La capacité des travailleurs à produire des écrits relatifs à leur travail et aux procédures employées est requise pour de nouvelles catégories qui, jusqu'alors, n'utilisaient pas l'écrit dans leur travail. Elle est également requise pour des catégories qui utilisaient l'écrit jusqu'ici, mais elle est requise sur de nouveaux sujets d'écriture. 

que ces procédures génèrent, ainsi que les sujets d'écriture sur lesquels l'auteur écrit, mais également, cela est tout aussi important, les sujets sur lesquels il n'écrit pas, en dépit des possibilités qui sont formellement offertes. On peut comprendre les rapports entre position d'écriture, auteur (individuel ou collectif) qui l'occupe et sujets d'écriture en intégrant à l'analyse les destinataires réels et potentiels de l'écrit, les enjeux qui se négocient dans leurs relations. l'observation directe, si possible, du travail et des conditions dans lesquels ces écrits spécifiques sont produits par les auteurs et reçus par les destinataires. Des entretiens, portants sur ces écrits, complètent l'approche qui ne peut se limiter à ces textes seuls. En effet, une grande part de l'information n'est accessible qu'à l'oral, en particulier celle relative à ce qui ne s'écrit pas ou à ce que les auteurs refusent d'écrire. « sortir » du cadre de l'entreprise particulière où l'enquête se déroule, de les resituer dans leur cadre pertinent qui est celui des rapports conflictuels entre des groupes sociaux qui ont des positions et des intérêts différents, des rationalités et des représentations concurrentes et qui luttent pour imposer ou défendre leurs intérêts, tels qu'ils se les représentent.

60 L'analyse des écrits professionnels que nous proposons montre les limites de l'efficacité de l'écriture du travail et des procédures, même quand les conditions peuvent sembler les plus favorables. Dans le cas étudié ici, les auteurs sont volontaires pour participer à l'amélioration de l'organisation et de l'efficacité de l'entreprise. Ils sont volontaires pour occuper la position d'écriture que le cercle crée, volontaires pour écrire les dysfonctionnements, rechercher et écrire de nouvelles procédures, sur lesquels tous les destinataires réels des comptes rendus peuvent être d'accord. On voit la complexité des enjeux sociaux que l'écriture du travail, par ces auteurs-là, met en œuvre et qu'elle conditionne les sujets sur lesquels on écrit, la manière dont on écrit et que cette écriture ne garantit pas une meilleure efficacité productive à la fin du processus.

61 Dans les procédures actuelles de certification, l'écriture du travail, des procédures et de leur application à un caractère obligatoire. On peut douter de leur plus grande efficacité et craindre qu'elles se limitent à des procédures qui prétendent augmenter l'efficacité des entreprises en rendant transparentes ${ }^{26}$ lisibles à proprement parler, les relations sociales qui se nouent à l'occasion du travail et qui, pourtant, concourent à la production et à son efficacité réelle. La lisibilité des relations sociales recherchée risque de correspondre aux capacités de lecture et de compréhension de ceux qui en sont les promoteurs. Quand cette recherche de lisibilité se croise avec la conception dominante aujourd'hui de l'entreprise (Le Goff, 1992) dans le monde des « managers », avec la conception de l'efficacité analysée par Jacques Le Mouël ${ }^{27}$, elle génère, sans doute, plus de conflits, de dysfonctionnements que de réelle efficacité sociale et économique. 


\section{BIBLIOGRAPHIE}

AFCERQ, (février 1984), Cercles de Qualité. Bulletin de l'AFCERQ, n 9.

Bourdieu, P., (1980), «Quelques propriétés des champs », in Questions de sociologie, Minuit, p. 113-120.

Bourdieu, P., (1992), Réponses, Seuil.

Bourdieu, P., (1994), « Pour une sciences des œuvres », Raisons pratiques, Seuil, p. 59-80.

Buron, B., (1989), « La parole au travail. Quand les travailleurs verbalisent leurs savoirs », Cahiers du LAST, n 5, Université de Tours, p. 75-100.

Buron, B., (1992), Une idéologie du marché et du consensus au travail. Nouvelle division du travail, modèle d'emploi et conflits dans la théorie du management participatif, Doctorat de sociologie, Groupe de sociologie du travail, Université Paris 7.

Chabaud, C. et De Terssac, G., (1987), « Du marbre à l'écran : rigidité des prescriptions et régulations de l'allure de travail », Sociologie du travail, $\mathrm{n}^{\circ}$ 3, p. 305-322.

Crozier, M., (1963), Le phénomène bureaucratique, Seuil.

Détienne, M. et Vernant, J.P., (1974), Les ruses de l'intelligence. La mètis des Grecs, Flammarion.

Fraenkel, B., (1991), La signature, Gallimard.

Goody, J., (1977), La raison graphique. La domestication de la pensée sauvage, Minuit. Avant Propos de Bazin, J., et Bensa, A.

Le Goff, J.P., (1992), Le mythe de l'entreprise, La Découverte.

Le Mouel, J., (1991), Critique de l'efficacité, Seuil.

Levi-Strauss, C., (1955), Tristes Tropiques, Plon.

de Ligny, G., (1982), Pratiques des cercles de qualité, Editions Hommes et Techniques.

Pave, F., (1989), L'illusion informaticienne, L'Harmattan.

Pividal, R., (1976), La maison d'écriture, Seuil.

Raveleau, G., (1983), Les Cercles de Qualité français, Entreprise Moderne d'Edition.

Segrestin, D., (1980), « Les communautés pertinentes de l'action collective », Revue française de sociologie, $\mathrm{n}^{\circ} 2$, p. 171-203.

\section{ANNEXES}

Pour illustrer la manière dont l'auteur écrit, en fonction de son sujet d'écriture et de sa position dans l'entreprise, nous donnons ici ${ }^{28}$ de larges extraits des comptes rendus des réunions d'un cercle de qualité sur une période de plus d'un an. Nommé Yakaémor, ce cercle est composé d'ouvrières non qualifiées issues du même atelier. Le chef d'atelier en a été l'animateur pendant près de 4 ans, bien qu'il soit considéré comme très directif, voire autoritaire par le personnel, y compris par les 
volontaires qui participent au cercle. Le nom que les membres ont donné au cercle traduit bien les rapports ambigus qui lient l'espace-temps de travail de l'atelier et sa hiérarchie à l'espace-temps du cercle. Nommer l'auteur Yakaémor, ou yaka est mort, c'est signifier que les formes d'exercice de l'autorité dans l'atelier ne sont plus pertinentes dans le cercle, c'est aussi et surtout le signifier devant celui qui les représente et les incarne au quotidien dans l'atelier. Au moment où les extraits commencent, le chef d'atelier vient d'être remplacé, à sa demande, dans son rôle d'animateur par un technicien de l'atelier. Ce changement provoque ou révèle une crise dans le groupe qui se désagrège et qui, en conséquence, perd sa qualité d'auteur parce qu'il n'a plus de sujets d'écriture.

77ème réunion, 11 février.

« La réunion commença par un tour de table qui n'apporta pas de nouvelles idées à

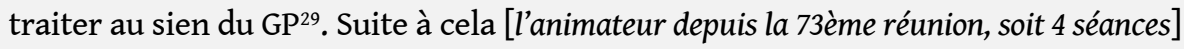
nous annonça la décision de Mme G. de se retirer temporairement du GP pour des raisons personnelles et nous fit part de son inquiétude de voir l'hémorragie s'aggraver suite à l'opinion de certaines personnes qui envisagent aussi leur retrait. Il proposa donc au groupe de suspendre l'étude en cours pour évoquer immédiatement ce problème avant qu'il ne s'aggrave. Il soumit cette idée au groupe qui l'adopta à l'unanimité. Un tour de table fut réalisé et il apparaît que les problèmes sont extérieurs en grande partie au GP. Le groupe prit la décision de le traiter comme une étude au sein du GP et de définir ainsi le sujet « Ambiance atelier et démobilisation GP ».

Nous commençâmes par un brainstorming pour essayer de définir toutes les causes à ce malaise général en précisant bien qu'il n'y avait en cela aucune critique

personnelle mais seulement une critique constructive dans un but positif pour amener le dialogue.

Liste des idées émises lors du brainstorming (dans l'ordre d'évocation et sans classement) : relations avec le chef d'atelier ; connaître les temps pour plus de motivation ; possibilité d'avoir la monitrice pour suivre des produits nouveaux ; savoir s'il faut travailler avec les plans, seule, ou avec l'aide de la monitrice ; soucis de travail qui préoccupent l'esprit ; meilleure compréhension entre préparatrices et mécaniciennes ; travail propre à chacun ; chacun répare ses propres défauts [ce qui entraîne une] prise de conscience ; changements fréquents de machine et de travail ; pas assez de machines ; les GP n'aboutissent pas ; les clans qui divisent l'atelier ; besoin de s'expliquer.

Suite à cela, le GP souhaiterait connaître l'avis des autres personnes de l'atelier afin d'étendre le débat à toutes les personnes désireuses de faire avancer la situation. [ L'animateur] clôtura la séance en expliquant à nouveau l'importance du GP et son rôle qu'il doit jouer dans la qualité de vie au travail en général et face aux problèmes existants, la solution n'était pas de baisser les bras et renoncer mais au contraire renforcer notre action au sein du groupe qui représente une issue pour lutter contre les nombreux problèmes qui subsistent. »

[Le coordonnateur commente, par écrit sur le compte rendu]

«Le GP a sans aucun doute choisi la meilleure option pour résoudre ce problème qui met en cause sa survie. La qualité de l'ambiance de travail fait en effet partie des problèmes que peut aborder un cercle de qualité. Attention de bien tenir compte de tous les avis pour résoudre ce problème!! »

[Le Directeur donne son accord, par écrit sur le compte rendu] 
« OK. Je participerai partiellement à la prochaine séance pour vous aider et apporter mon appui à un bon redémarrage. »

78 ème réunion, 26 février.

«La réunion commença par l'intervention [du Directeur] venu nous apporter son soutien pour la bonne marche de notre GP et nous encourager à poursuivre cette étude qui, correctement entreprise, serait très positive pour l'ensemble de l'atelier. [Le coordonnateur ] était également présent pour nous apporter son aide et tous deux nous ont précisé qu'ils étaient prêts à venir dialoguer si le GP en exprimait le besoin.

Suite à ce dialogue positif encourageant pour la suite de notre étude, le dernier quart d'heure fut employé à la préparation de la prochaine réunion et au choix de notre salle GP. Nous avons le choix entre les salles $n^{\circ} 2$ et $n^{\circ} 3(. .$.$) et le groupe choisit la salle n^{\circ} 3$ à l'unanimité. [Le coordonnateur] nous expliqua que nous avions la possibilité d'aménager cette salle avec notre budget GP.

Ordre du jour de la prochaine séance : définir ensemble les modalités de notre étude et comment recueillir l'avis des différentes personnes concernées à l'atelier ; plusieurs possibilités s'offrent à nous pour cela.

Notons la présence de Mme G. revenue se joindre à nous pour cette étude, motivée par le problème, illustrant en cela le réel besoin ressenti dans l'atelier et nous confortant dans le choix du sujet traité. »

80 ème réunion, 25 mars.

«Comme prévu durant la dernière réunion, [l'animateur] distribua quelques jours auparavant à tous les membres permanents de l'atelier les feuilles pour le sondage. Un quart d'heure avant la réunion celui-ci collecta les bulletins sous enveloppes cachetées et anonymes (seules les personnes qui le désiraient avaient la possibilité de faire figurer leur nom sur le bulletin). Il est à noter que tout le monde remit son bulletin.

La réunion commença par un petit brainstorming mais il ressort que c'est surtout l'impatience du dépouillement des bulletins qui domine. Mme D. fut volontaire pour noter les idées principales au tableau pendant que [l'animateur] lisait les réponses à haute voix de façon à ce qu'il n'y ait pas de reconnaissance éventuelle des écritures.

L'assistance fut très attentive et réfléchie tout en relevant les idées maîtresses. Certaines feuilles étaient vierges signées ou non et le groupe se découragea quelque peu à ces moments ; d'autres pensaient que le sujet est très valable mais doutaient de l'issue et ce sentiment serait devenu contagieux sans les efforts de l'animateur. Par contre, beaucoup de réponses furent très positives et enrichissantes; l'esprit de l'étude étant très bien perçu et il apparaît que pour certains la feuille de sondage n'était pas suffisante pour exprimer leurs sentiments et idées avec beaucoup de philosophie.

Afin de terminer le dépouillement et de ne pas garder d'enveloppes en attente, le groupe décida à l'unanimité de poursuivre encore un quart d'heure après midi sonné, prouvant ainsi son attachement à cette étude. Les bulletins furent détruis par [l'animateur] qui garda ainsi sa parole vis à vis de l'anonymat des personnes. (...) Souhait du groupe pas de diffusion de ce rapport. "

81ème réunion, 22 avril.

«Cette réunion se déroula avec un effectif très réduit dû à l'absentéisme causé par la maladie ou les congés. Nous étions trois au total mais la réunion eu lieu malgré tout. En effet, la dernière séance s'effectua au complet et fut très enrichissante de par les idées émises lors du sondage ; la preuve fut que les membres donnèrent une dizaine de minutes 
de leur temps pour achever le dépouillement.

Les idées et les réponses aux questionnaires étaient notées sur le tableau mais il restait à les classer en recherchant les facteurs principaux. Nous décidions de commencer le classement, ce qui ne portait pas préjudice aux absents mais permettrait d'avancer. D'après le sondage, il ressort quatre familles de causes, qui sont : DIALOGUE ; COMPORTEMENT ; INFORMATION ; ORGANISATION. Nous avons commencé à classer les causes dans ces quatre rubriques et cela se vérifie très nettement (...). Souhait du groupe : ne pas diffuser le compte rendu. »

89ème réunion, 22 septembre.

"Après avoir fait un tour de table où il n'a été évoqué que des problèmes de méthodes à traiter ponctuellement, le groupe a commencé l'élaboration du diagramme cause-effet afin de clarifier tous les résultats obtenus lors de nos enquêtes et débats.

Lors de la séance prochaine nous achèverons ce diagramme et définirons nos critères afin de rentrer dans la phase « active" de notre étude pour la recherche des solutions. »

91ème réunion, 21 octobre.

" [L'analyse] fut poursuivie dans l'esprit d'objectivité en tenant compte des remarques et des sondages effectués. Certains problèmes ont évolué positivement par la volonté de tous et cela sera évoqué lors du choix des solutions envisagées. Ex : création d'un 2ème GP ; souhait des anniversaires ; réunions atelier. Toutes les idées seront acceptées venant des membres de l'atelier pour compléter ce tableau si besoin.

Prochaine séance : choix des critères ; recherche des solutions en choisissant une des cinq grandes familles de problèmes à traiter en priorité. »

92ème réunion, 2 novembre.

"La séance fut consacrée au suivi de notre étude pour que celle-ci soit suffisamment avancée pour la présentation au séminaire ${ }^{30}$ sans toutefois adopter une précipitation néfaste aux résultats.

Recherche des critères : ne léser personne; en accord avec la hiérarchie; solution adoptée à l'unanimité ; ne pas gêner la production.

Puis nous avons fait une analyse branche par branche du diagramme causes-effets.

I. L'information: principe d'une réunion mensuelle avec l'atelier et [le chef d'atelier] (définie lors des entretiens du GP avec [le chef d'atelier], 2 réunions ont déjà eu lieu) ; boite pour déposer les questions à développer lors des réunions atelier; réunion extraordinaire en cas de nécessité ; affichage des objectifs à réaliser dans le mois avec les délais et les temps; problème de la pause à définir lors d'une réunion avec [le chef d'atelier] ; donner résumé du compte rendu des réunions de productivité ; rapport de contrôle communiqué à l'exécutante pour s'autocorriger ; développer les fiches de communication dans l'atelier. II. La formation: chaque personne explique son travail avec les difficultés qu'elle rencontre (ex. pliage) ce qui entraîne une prise de conscience des points particuliers à surveiller ; désir de connaissance de ce que fait l'autre personne ; désir de connaissance des différents éléments du siège (ré-explication le cas échéant, surtout pour les personnes nouvelles) éventuellement réalisation d'une cassette vidéo ou d'un fascicule avec photos ; désir d'information sur les réalisations des autres ateliers (ex. visite) ; l'explication du tableau de bord a été faite lors de la dernière réunion atelier avec [le chef d'atelier]; tout le monde devrait suivre la formation réglage machine ; formation GP en cours à élargir à tous les membres de l'atelier pour s'imprégner de l'esprit GP. (...) » 


\section{NOTES}

1. L'écriture des procédures réelles ainsi que le contrôle écrit de la manière dont elles sont appliquées au quotidien occupent une place centrale dans la conception actuelle de la qualité.

2. Peut-on imaginer une théorie d'organisation qui aurait des objectifs inverses?

3. Selon des modalités qui peuvent varier suivant les entreprises.

4. R. Pividal (1976) attire notre attention sur le rapport particulier que l'on établit entre écriture et vérité. La parole, dit-il, «n'est, dans notre société, jamais en rapport avec la vérité, et la preuve : la science ne se sert que de l'écriture. » Si ce rapport spécifique est dominant, il n'est pourtant pas général dans tous les groupes qui composent la société française aujourd'hui. On pensera, par exemple, aux relations entre acheteurs et vendeurs sur certains marchés, tels les maquignons... Dans ce cas, l'accord est scellé oralement et lie les contractants avec la même force qu'un contrat écrit.

5. B. Fraenkel (1991), montre que la signature transforme le statut du texte. La notion de droit d'auteur en France considère l'œuvre comme émanation de la personne de l'auteur, ainsi, l'œuvre ou le texte signé accède à un statut immatériel et est inaliénable. De toute évidence, dans les écrits professionnels, la notion de droit d'auteur ne s'applique pas. De ce point de vue, l'auteur d'écrits professionnels dans les ces procédures ne semble pas avoir de droit, mais uniquement des devoirs ou des responsabilités.

6. Au sens de vouloir dire, mais aussi de pouvoir dire.

7. Au sens où l'entend Norbert Elias.

8. Josiane Boutet a justement soulevé ce point lors du 1er Séminaire CNAM-GERICO, Paris, 26 novembre 1993. La position de C. Lévi- Strauss (1955, p. 344, pour qui « la fonction primaire de la communication écrite est de faciliter l'asservissement » nous parait relever de la même tendance à la projection, bien que cette projection aille dans un sens radicalement opposé.

9. Pour P. Bourdieu, 1992, Réponses, Éditions du Seuil, p. 72-73, un champ "peut être défini comme un réseau, ou une configuration de relations objectives entre des positions. Ces positions sont définies objectivement dans leur existence et dans les déterminations qu'elles imposent à leurs occupants, (...) par leur situation actuelle et potentielle dans la structure de la distribution des différentes espèces de pouvoir (ou de capital) dont la possession commande l'accès aux profits spécifiques qui sont en jeu dans le champ... ».

10. Tous les textes qui la régissent ne sont pas produits dans l'entreprise. Certains relèvent du cadre réglementaire, pour la sécurité par exemple, ou contractuel comme les conventions collectives. Tous ces textes sont subordonnés à l'ordre légal qui se manifeste dans le cas du contrat de travail, que l'on peut considérer comme « texte » central.

11. Pour l'analyse stratégique de M. Crozier (1963), le détournement des règles de l'organisation, sous réserve que ce détournement reste caché, crée des zones d'autonomie qui renforcent la position dans les jeux de pouvoir des acteurs qui les contrôlent.

12. C. Chabaud et G. De Terssac (1987) montrent que, dans la presse quotidienne, les opératrices interprètent les règles formelles de productivité en fonction de normes implicites et que ce détournement est une des conditions qui permet au journal de sortir à temps chaque jour. Les auteurs affirment que la « contestation des prescriptions formelles obéit en fait à une logique de l'efficacité, que clandestin et productif vont souvent de pair.»

13. Seule une conception naïve du monde du travail pourrait empêcher le chercheur de voir la réalité des conflits de pouvoir, d'intérêts et de représentation qui traversent aussi la structure hiérarchique des entreprises. Quand des cadres s'opposent aux cercles de qualité, ces oppositions ne se manifestent jamais ouvertement, il s'agit tout au plus de formes d'inertie, de rétention de l'information. 
14. On rencontre quelques cas où aucun responsable hiérarchique de l'atelier ou du service n'est pas volontaire pour participer au cercle. L'animation est alors assurée par l'un quelconque des volontaires, désigné par ses pairs.

15. En français, le remue-méninges.

16. Cette technique peut être très efficace. Certains cercles recensent plus d'une centaine de dysfonctionnements dans leur atelier.

17. Le monde managérial semble croire aux vertus des « outils».

18. Il s'agit d'éviter qu'un cercle choisisse comme sujet d'écriture un problème que la hiérarchie de l'atelier ou les services techniques sont déjà en train de résoudre. Il permet au coordonnateur d'intervenir pour aider un cercle, si, par exemple, un chef de service refuse d'apporter sa collaboration au groupe. Il s'agit aussi de faire circuler l'information technique, de faire connaître les solutions techniques qui, découvertes par un cercle, peuvent être généralisées.

19. J. Bazin et A. Bensa, "Avant-propos », in J. Goody, (1977, p. 13).

20. Le devise complète du cercle est : Les 3 Mousquetons, une équipe qui s'accroche.

21. Compte-rendu, cercle Boîte à Idées, 6ème réunion.

22. Compte-rendu, cercle Oxygène, 33ème réunion.

23. Compte-rendu, cercle Oxygène, 35 ème réunion.

24. Une politique inverse, pour réduire le volume des prestations versées dans une période où les régimes de protection sociale sont déficitaires, constituerait non seulement un manquement à la loi, mais aussi une atteinte à l'esprit de la Mutualité.

25. Pour le personnel, ces deux fonctions sont justifiées abstraitement au nom de la solidarité. A ce titre, il est normal que les actifs paient les cotisations légales, il est également normal que ceux qui refusent de payer soient poursuivis en procédure contentieuse.

26. F. Pavé, 1989, L'illusion informaticienne, Paris L'Harmattan, montre que l'informatisation de la production repose sur l'illusion que l'efficience des organisations repose sur la transparence des relations sociales.

27. J. Le Mouël, 1991, Critique de l'efficacité, Paris, Éditions du Seuil, montre que le modèle d'efficacité managériale, diffusé par les consultants, repose souvent sur le sophisme suivant : «Ce qui est efficace est vrai. Or, ce qui est vrai est juste. Donc, ce qui est efficace est juste. », p. 14.

28. La qualité des documents originaux ne permettait pas une simple reproduction. En revanche, les paragraphes et les soulignements sont originaux. Les coupures sont signalées par (...) ; les parties en italiques et entre crochets [ ] ont été rajoutées par moi, B. BURON.

29. Dans l'entreprise, les cercles de qualité sont appelés groupes de progrès (GP).

30. Chaque année, à la mi-novembre, tout le personnel qui participe aux GP est réunit pour présenter les résultats obtenus.

\section{RÉSUMÉS}

L'auteur propose d'analyser les écrits professionnels comme des oeuvres produites par des auteurs qui occupent une position spécifique dans le champ de production des écrits professionnels dans les entreprises. A partir des comptes-rendus de réunions de cercles de qualité, il montre les nouvelles positions d'écriture qui se créent, comment de nouvelles catégories d'auteurs se constituent et occupent ces positions, la manière dont ils écrivent sur les nouveaux sujets d'écriture que ces procédures autorisent, comment ces écrits sont reçus par 
leurs destinataires mais aussi comment des auteurs peuvent refuser d'écrire sur certains sujets. L'analyse montre la pluralité des enjeux sociaux qui se négocient autour de ces opérations d'écriture du travail et des procédures, qui ne se réduisent jamais à des opérations techniques. En ce sens, les refus d'écriture, ou les difficultés que les travailleurs rencontrent pour écrire leur travail dans les procédures de certification qui se développent, tiennent moins à de supposés «manques de formation » qu'à la nature même du processus en cours. Si l'écriture du travail vise aussi à le rendre « lisible » à l'extérieur de l'entreprise, par les clients notamment, on peut se demander si tous les « destinataires » ont bien les mêmes critères de lisibilité.

The author intends to analyse professional writings as works produced by authors occupying a specific spot in the field of production of professional writings within the firms. Investigating the minutes of the meetings of the "cercles de qualité ", he points out: (1) the categorization process of various authors, differing from each others in the manner chosen to write about such new issues allowed by these institutions, (2) how these writings are received by their recipients, (3) how some authors happen to refuse to write about some topics.

So the article describes the variety of social stakes negociated aroud such writing process, and the variety of difficulties faced by writers as well as readers of the new writing products referring to emerging procedures in the field of contemporary work.

\section{INDEX}

Keywords : professional writing, quality approach, working conditions, work organization, management, author

Mots-clés : écriture professionnelle, démarche qualité, conditions de travail, organisation du travail, management, auteur

\section{AUTEUR}

\section{BERNARD BURON}

Bernard Buron, LAST (Laboratoire d'Anthropologie et de Sociologie de Tours) 